\title{
Recuperación de la tradición textil en Santa Perpètua de Mogoda: una aproximación desde la práctica artística en clave de Aprendizaje-Servicio
}

\author{
Recovery of the textile tradition in Santa Perpètua de Mogoda: an \\ approach from artistic practice through Service-Learning
}

\author{
Anaïs Civit López ${ }^{1}$ \\ ${ }^{1}$ Universitat de Barcelona. anaiscivit@ub.edu
}

Recibido: 30/6/2021

Aceptado: 18/10/2021

Copyright (C)

Facultad de CC. de la Educación y Deporte.

Universidad de Vigo

\section{Dirección de contacto:}

Anaïs Civit López

Departamento de Artes y Conservación-

Restauración

Facultat de Belles Arts

Universitat de Barcelona

Pau Gargallo, 4

08028 Barcelona

\begin{abstract}
Resumen
Memòria, recuperació d'artesania $i$ sostenibilitat es un proyecto entre la Universitat de Barcelona y los IES Rovira-Forns y Estela Ibèrica de Santa Perpètua de Mogoda. Pretende recuperar los sistemas productivos tradicionales de la artesanía aplicándolos a la producción artística y al diseño; dotar al alumnado de las herramientas necesarias para la producción artesanal vinculada con la memoria histórica local; intercambiar con la población las costumbres y tradiciones en relación con la producción artesanal; evidenciar, basándose en los preceptos fundamentales de la economía feminista, la importancia del trabajo doméstico. El proyecto se desarrolla a través de la metodología de Aprendizaje-Servicio (ApS): estudiantes del Grado de Bellas Artes tutorizan a alumnado de tercero de ESO de los dos institutos a través del Programa de Servicio Comunitario del Ayuntamiento de Santa Perpètua de Mogoda. Mediante la ficha didáctica Identitats truncades (2020) de Eulàlia Grau Costa et al., en clave de metodología del descubrir, el alumnado explora la realidad de la producción textil y artística. El proyecto culmina con una primera exposición y unas jornadas de investigación y docencia artística. Se potencian valores sociales como la reutilización y un modelo textil más sostenible para incidir directamente en el territorio y la comunidad recuperando la rica memoria local textil.
\end{abstract}

\section{Palabras clave}

Aprendizaje-Servicio, Arte Textil, Transferencia de Conocimiento, Economía Feminista, Comunidad

\begin{abstract}
Memòria, recuperació d'artesania i Sostenibilitat is a project between the University of Barcelona and the Rovira-Forns and Estela Ibèrica of Santa Perpètua de Mogoda high schools. The project aims to recover the traditional production systems of crafts, applying them to artistic production and design; to equip students with the necessary tools for artisan production linked to local historical memory; to exchange customs and traditions with the population in relation to artisan production; to demonstrate,
\end{abstract}


based on the fundamental precepts of feminist economics, the importance of domestic work. The project is developed through the Service-Learning method: students of the Bachelor of Fine Arts tutor third-year ESO students from both institutes through the Community Service Program of the Santa Perpètua de Mogoda City Council. Through the didactic sheet Identitats truncades (2020) by Eulàlia Grau Costa et al., using Self-Learning method. Students explore the reality of textile and artistic production. The project culminates with a first exhibition and a journal of research and artistic teaching. Social values such as reuse and a more sustainable textile model are promoted to directly influence the territory and the community by recovering the rich local textile memory.

\section{Key Words}

Service-Learning, Textile Art, Transference of Knowledge, Feminist Economy, Community

\section{INTRODUCCIÓN}

Presentamos el proyecto Memòria, Recuperació de l'artesania i Sostenibilitat, una propuesta en clave de género a partir de la experimentación con los materiales textiles y los métodos de la creación artística. Esta propuesta es fruto de la colaboración entre la Facultad de Bellas Artes de la Universitat de Barcelona, concretamente el grupo ATESI, y los Institutos de Educación Secundaria (IES) Rovira-Forn y Estela Ibèrica de Santa Perpètua de Mogoda (Barcelona). Se presenta el desarrollo y los resultados del proyecto durante el curso 2020-2021, que ya se inició durante el curso 2019-2020 y que espera tener continuidad en los cursos próximos. Se pretende la valorización de la tradición cultural textil mediante la expresión artística, la sostenibilidad y el reciclaje obteniendo patrones de piezas textiles con memoria de usos y anécdotas, a partir de las que trabajar su descontextualización y construir un relato colectivo desde la empatía, desde valores simbólicos y miradas hacia la transformación social. Además, se enfatiza la valorización de las actitudes participativas y de las metodologías de la creación como elementos de transferencia del conocimiento, de transformación social, de resiliencia y de memoria viva.

Es evidente la preocupación desde las disciplinas artísticas por el cambio en las actitudes y los marcos conceptuales en todo aquello referente al género y a la sostenibilidad. Es, por lo tanto, de notorio interés un proyecto que aboga por el cambio en las mentalidades del alumnado tanto del ámbito universitario como de Educación Secundaria Obligatoria, fomentando patrones y actitudes en clave de género y en clave de producción sostenible. La creación artística no puede ser ajena a los cambios que socialmente se producen en el debate colectivo y debe afrontarlos no sólo como una consecuencia de dichos cambios, sino como un agente de transformación con voz propia, fomentando el debate y la participación de todos los miembros de la sociedad. En este sentido, el proyecto que presentamos aboga por el trabajo artístico alrededor de la recuperación de la tradición textil, la sostenibilidad en los medios de producción, el debate en clave de género, la economía feminista, etc.

Con las fechas reivindicativas del 25 de noviembre (Día Internacional de la Eliminación de la Violencia contra la Mujer) y del 8 de marzo (Día Internacional de la Mujer) como trasfondo, se ha construido la actividad Identitats truncades a través de una ficha didáctica en clave de metodología del descubrir, junto con un soporte 
videográfico que flexibiliza la propuesta más allá de la presencialidad, dada la incertidumbre por el Covid-19.

\section{METODOLOGÍA}

\subsection{Aprendizaje-Servicio}

El Aprendizaje-Servicio (ApS) es un método educativo que combina procesos de aprendizaje y un servicio a la comunidad articulados en un único proyecto (Rubio et al., 2014). De este modo, los participantes del proyecto aumentan la calidad de su aprendizaje a medida que aplican lo que ya saben o lo que han aprendido en un entorno de necesidad real que les exige poner en práctica los contenidos del contexto en que se ha planteado el proyecto. El ApS implica, por lo tanto, una filosofía de la educación que no se basa en la acumulación de méritos curriculares, sino en la concepción del aprendiz como un ente social capaz de influir directamente en la sociedad que lo rodea y de convertirse en un agente de cambio. Además, se ha escrito largamente sobre los beneficios educativos de aplicar lo aprendido a un contexto de servicio social (Deeley, 2016; Puig, 2012; Escofet y Rubio, 2017). Dichos beneficios pasan por la evidencia de que un contenido conceptual o competencial dado en un contexto académico se consolida más fácilmente cuando el marco en que se puede aplicar no es sólo teórico, sino también práctico. Es decir, cuando un aprendizaje puede utilizarse para una realización cotidiana, para un servicio a la comunidad, dicho aprendizaje se consolida de forma mucho mayor (Deeley, 2016). Además, la complementación entre el aprendizaje y el servicio implica el trabajo necesario de múltiples competencias del ámbito social y personal que se relacionan con el servicio comunitario. Cabe mencionar los Objetivos de Desarrollo Sostenible de la Agenda 2030, que implican una pedagogía de la responsabilidad cívica, un compromiso político de la Universidad y de los agentes que forman parte, las competencias que se relacionan con el bien común y los beneficios sociales de una acción comunitaria.

La Universidad de Barcelona dispone, hoy en día, de un grupo de fomento del Aprendizaje-Servicio formado por profesorado de distintas facultades, cuyos objetivos pasan por la integración de la Universidad en el espacio educativo público. Algunas de las múltiples facultades que participan en este grupo son la de Bellas Artes, Biología, Ciencias de la Tierra, Derecho, Economía y Empresa, Educación, Trabajo Social, Filosofía, Física, Geografía e Historia, Psicología, Matemáticas, Informática, etc. Desde la Facultad de Bellas Artes se trabaja con la perspectiva de la creación artística como un bien que repercute en la comunidad y que, por lo tanto, es susceptible de trabajar múltiples competencias que se relacionan con el Aprendizaje-Servicio.

En el caso que nos ocupa, el proyecto se hilvana a través del Aprendizaje-Servicio dada la acción tutorial en un doble sentido. Por un lado, el profesorado de la Facultad de Bellas Artes tutoriza los alumnos de la asignatura optativa UEA Nous Recobriments $i$ Continents en Art de tercer y cuarto curso del Grado de Bellas Artes. A su vez, el alumnado de la Facultad realiza una tarea de tutorización hacia el alumnado de los Institutos de Educación Secundaria Rovira-Forns y Estela Ibèrica, ambos de Santa Perpètua de Mogoda. De esta forma, el alumnado de la Facultad realiza una tarea eminentemente social relacionada con la necesidad del estudiantado de Secundaria de 
disponer de un arte más a su alcance y más cercano a su realidad. Al mismo tiempo, el alumnado de los centros de Educación Secundaria desarrolla sus capacidades creativas y las ofrece a la comunidad, revalorizando la tradición textil de la población, promoviendo valores de cultura sostenible y de economía doméstica en clave de género. El proyecto implica, por lo tanto, un doble trabajo por parte del alumnado que los lleva a consolidar sus conocimientos aplicándolos a la enseñanza secundaria y a la producción artística. El alumnado de los centros de Educación Secundaria participa en el proyecto a través de su programa de servicio comunitario.

El proyecto, además, es un buen ejemplo de transversalidad curricular y experiencia de servicio comunitario, participando de las siguientes áreas:

- Educación Visual y Plástica: diseño y creación de las obras, composición de espacios creativos y expositivos;

- Lengua Catalana: redacción de los textos vinculados a las obras;

- Física y Química: conocimientos técnicos y procedimentales vinculados a la producción de tintes naturales y los procedimientos textiles;

- Ciencias de la Naturaleza: producción y extracción de materias primas;

- Ciencias Sociales: artesanía y tradición textil, memoria histórica local;

- Cultura y Valores: arte comunitario, sostenibilidad, reciclaje, inclusión y trabajo de género;

- Matemáticas: dimensiones, medidas y trabajo a escala.

\subsection{De lo social en lo textil}

Trabajar un proyecto con un fuerte componente social implica tener presentes cuáles son las cuestiones sobre las que se quiere incidir directa o indirectamente. En este caso, a través del trabajo textil nos proponíamos exponer cuestiones que conciernen directamente al alumnado tanto de Secundaria como del ámbito universitario como son las reflexiones en torno a la sostenibilidad, la identidad de género y sexual, $\mathrm{y}$ reflexiones que atañen al papel de las mujeres en el desarrollo socioeconómico a través de su aportación que, en muchas ocasiones, se ampara en el marco de la economía informal.

Incidiendo precisamente en este último punto, debemos hablar del cambio de perspectiva que impulsan desde inicios de los años sesenta y setenta del siglo XX las teorías de la economía feminista. Dichas teorías se basan en la reformulación de los postulados de la economía tradicional para anteponer el cuidado de la vida y del planeta a la producción y la acumulación de capital (Carrasco, 2014). No se trata, pues, de una inclusión de las mujeres en la economía tradicional, sino de un cambio de paradigma radical, que permite construir una disciplina que analice no sólo el mercado y los aspectos bursátiles o fiduciarios, sino también toda aquella actividad que permite el funcionamiento de una sociedad y, especialmente, que permite el bienestar de sus miembros y el equilibrio ecológico. Así pues, la economía feminista, según la perspectiva de Carrasco, no es una teoría única, sino un conjunto de posicionamientos económicos que tienen que ver con la propuesta de cambio político, donde la vida de las personas sea el centro de la disciplina social y su bienestar el objetivo económico de todo marco teórico. En resumen, pensar la economía desde una perspectiva feminista conlleva una serie de cambios drásticos de valores, un cambio de los medios de 
producción y los tiempos, cambios en la vida cotidiana y una nueva estructura de consumo.

Entender y revalorizar las técnicas artísticas y productivas nos lleva a incluir valores de sostenibilidad implícitos en aquello que deriva de un ritmo de vida más orgánico y del cuidado del hogar, de las personas y del medio. El proyecto trabaja con la idea de una producción y un consumo sostenible, implementa prácticas de reutilización, trabajo con fibras y tintas naturales en oposición al sistema industrial textil que supone actualmente uno de los sectores productivos más contaminantes, especialmente por el uso de tintes y colorantes sintéticos (Cortázar Martínez et al., 2014).

A través del conocimiento textil y la práctica de tareas desarrolladas tradicionalmente por las mujeres, se trasladan al alumnado estas reflexiones para evidenciar la desigualdad y el menosprecio hacia las tareas domésticas y, en definitiva, hacia todo aquello necesario para el cuidado de la vida que se traduce en una falta de remuneración (valor económico) y de reconocimiento (valor social). Es un proyecto, por lo tanto, que ofrece la posibilidad de trabajar mediante lenguajes artísticos variados para reflexionar, visibilizar y transformar aspectos que forman parte de las inquietudes reales de la ciudadanía y el estudiantado.

De nuevo, se delega este cuidado (hacia el planeta) a las mujeres, con soluciones que no implican una profesionalización y acceso a empleos relacionados con la sostenibilidad, sino que forman parte de sus supuestas tareas diarias. En palabras de Alicia Puleo:

"Con el surgimiento de las preocupaciones ecológicas, algunos han invitado al tradicional "ángel del hogar" a convertirse en salvador del planeta. Esta solución es inoperante. Pero además, es "impertinente", como ha sugerido Amelia Valcárcel. No se debe pedir a las mujeres que sean las principales cuidadoras del medio ambiente. [...] no se puede hacer politica ambiental a costa de las mujeres, es decir, favoreciendo los papeles tradicionales" (Puleo, 2011, pp. 20-21).

Para ello, se pide al alumnado que extraiga patrones de diversos objetos y, a través de estos, generen piezas textiles con nuevos significados, recalcando así el valor artístico de los procesos productivos tradicionales y propios de la economía informal con unas fechas reivindicativas concretas (el 25 de noviembre, Día Internacional de la Eliminación de la Violencia contra la Mujer; y el 8 de marzo, Día Internacional de la Mujer) que sirven como motivo. El proyecto, pues, pretende reflexionar a través del arte textil alrededor de estas cuestiones y poner en valor aquello textil como medio de expresión artística, promoviendo la creación en un entorno cotidiano y la producción artesanal como un medio más de producción de arte. Es decir, revalorizar las tareas cotidianas que han sido siempre necesarias, pero a las que no se les ha dado el valor que tienen otras tareas no tan significativas para el cuidado de la vida.

\subsection{Metodología del descubrir}

El proyecto se articula a través de una ficha pedagógica titulada Identitats truncades (Grau Costa et al., 2020) a partir de la cual se articulan todos los procesos de aprendizaje y supone el eje central de las acciones de creación (ver Figura 1).

Para comprender el alcance de la propuesta y los procesos de aprendizaje y desarrollo de las capacidades del alumnado participante debemos comprender primero la metodología de trabajo y la labor pedagógica que hay detrás de todo ello. Desde hace 
ya algunas décadas, profesorado diverso de la Facultad de Bellas Artes de la Universidad de Barcelona y profesorado que ha estado en contacto directo, ha desarrollado una metodología propia de trabajo llamada metodologías del descubrir (Grau Costa, 2009, 2013; Porquer Rigo et al., 2015; Sasiain Camarero-Núñez, 2012). Tal y como dicen los propios autores:

"Aunque el concepto se acerca inevitablemente a los utilizados por Jerome Bruner o Jean Piaget entre otros por algunas de sus propuestas para la educación primaria (aprendizaje por descubrimiento, Inquiry Based Learning), las metodologías del descubrir deben entenderse en un contexto diferente en tanto que están pensadas especialmente para las etapas de educación secundaria y universitaria y en tanto que nuestras propuestas no dan especial prioridad ni a los conocimientos ni a la experimentación científica. Nuestras metodologías plantean la autocontemplación como investigación, donde la intuición es la herramienta preferente" (Grau Costa y Porquer Rigo, 2017, p.17).

A través de una ficha pedagógica se propone un enunciado concreto que incluye unas determinadas pautas y genera interrogantes más o menos explícitos y con un procedimiento, técnica o concepto concreto. Cada ficha se compone de los apartados siguientes: propuesta de trabajo, enunciado, para hacerlo, condicionantes, proceso, materiales, presentación y material de consulta. Esto que en un principio podría parecer simple, se convierte en una potente herramienta que apela directamente a los procesos de creación de aquel alumnado que lo recibe, genera dudas, admite una interrogación constante empezando por la propia ficha y plantea un reto personal para resolver el encargo concreto que se le plantea. La ficha se convierte en una "excusa" o "trampa" en la que el alumnado se ve inmerso en un proceso personal de desarrollo de estrategias para resolver la tarea donde debe enfrentarse a dificultades técnicas, de autoevaluación, de razonamiento y deducción y donde poner en valor su intuición.

Esto permite al profesorado articular la docencia a partir de una serie de fichas como eje de trabajo o bien introducirlas de manera puntual en una actividad formativa determinada. Las fichas permiten ajustes según la edad y en función del contexto o la necesidad.

"[...] no proponemos una ordenación programática que parta de unidades didácticas agrupadas por temáticas y de las cuales se desprendan fichas didácticas, sino que, invirtiendo el sistema, trabajamos con actividades didácticas en formato de ficha de forma que estas se ordenan y programan dentro de diferentes ritmos según la programación, la asignatura y el equipo docente" (Grau Costa, 2013, p.13).

Además, se relacionan directamente con las filosofías de la pedagogía colectiva y del arte social, así como las competencias propias del área artística:

"Su uso nos ha demostrado su potencial capacidad de sugestión y de motivación en la práctica; como una herramienta que se convierte en un eje vertebrador para un discurso continuado, un elemento que permite la interacción puntual entre un motivo escogido y una finalidad buscada. La ficha, en su simplicidad aparente, actúa como un anclaje de conocimiento orientativo para enlazar con un despliegue conceptual, procedimental y actitudinal para la formación/generación de creatividad y el desarrollo de competencias propias del área artística. Fomenta el autoaprendizaje. Podemos trabajar directamente procesos creativos y generar discursos de pensamiento individual que, fácilmente, escalan en procesos de debate colectivos -entroncando con la filosofía de las pedagogías colectivas o del arte social-" (Grau Costa y Porquer Rigo, 2017, p.17). 
Así la autora pone la intuición, la autocontemplación y el proceso en el centro de la creación artística. Por lo tanto, la metodología del descubrir aplicada a través de las fichas pedagógicas permite al alumnado abrir vías de exploración desconocidas explorando conocimientos ya adquiridos para ser capaces de desarrollar una tarea concreta sin ser conscientes de las potencialidades de aquello que parece que se les pide.

\section{EL PROYECTO}

\subsection{Objetivos}

- Dar a conocer iniciativas pedagógicas y artísticas que potencien la colaboración entre las universidades y las entidades del territorio para generar transferencia de conocimiento mediante acciones comunitarias.

- Promover proyectos artísticos sostenibles con valores sociales y éticos que tengan en cuenta las necesidades sociales reales del territorio.

- Intercambiar con la población usos y costumbres en relación con la producción artesanal textil, con el uso de procedimientos tradicionales de confección, patronaje artesanal y el uso de fibras y tintes naturales.

- Valorizar (desde la perspectiva de la economía feminista) el trabajo de las mujeres en el hogar o en la economía sumergida y la tradición textil evidenciando su importancia en la evolución socioeconómica e histórica local.

\subsection{Agentes implicados y descripción de la colaboración}

Para comprender el valor y la aportación de cada una de las partes implicadas en el proyecto, debemos describir brevemente su función y las colaboraciones que se establecen en el territorio.

Por un lado, el equipo docente, coordinado por Eulàlia Grau Costa, perteneciente al Grupo de Innovación Docente Consolidado ATESI GINDO-UB/162 como promotor del proyecto y generador de la propuesta. Realiza el seguimiento del proyecto, facilita el material y las herramientas necesarias (máquinas de coser, tejido, material de costura, etc.) y coordina las acciones formativas, de exposición, difusión y análisis de resultados. A partir de la metodología de Aprendizaje-Servicio (ApS) se oferta la participación en el proyecto al alumnado de tercer y cuarto curso de la asignatura optativa UEA Nous Recobriments $i$ Continents en Art del Grado de Bellas Artes. El proyecto se relaciona de manera natural con el currículo de la asignatura dado que esta se centra en los procedimientos textiles ligados a la escultura y aplica la metodología ApS como actividad formativa obligatoria de su programación. El alumnado del Grado, que recibe en esta asignatura una formación textil y en acciones comunitarias, realiza también el encargo de la ficha pedagógica y actúa tutorizando al alumnado de los IES. Un total de diez alumnas ha participado directamente en el proyecto. Cabe destacar que el papel de las alumnas-tutoras no es meramente el de acompañantes, sino que tienen una triple tarea. Por un lado, comparten sus conocimientos y realizan el soporte formativo tanto en cuestiones técnicas como de desarrollo conceptual y vehiculan las ideas que el alumnado de Secundaria quiere comunicar con sus piezas; comparten experiencias 
formativas e intereses durante el desarrollo del proyecto manteniendo un canal fluido y directo de comunicación (se ha optado por la comunicación mediante un grupo conjunto de mensajería); por último, como alumnas del Grado, realizan a su vez una pieza siguiendo los mismos preceptos que el alumnado de Secundaria.

Queremos hacer hincapié en la labor del grupo de docentes e investigadores que forma el grupo ATESI dado que los preceptos y las líneas de actuación están ligadas a los objetivos del proyecto que nos ocupa. Se trata de un grupo de Innovación Docente que centra su investigación y actividad en la incidencia directa en los sectores educativos y sociales del territorio apostando por el acercamiento desde el arte social a necesidades de intercambios con colectivos y entidades respondiendo a necesidades reales. Se entiende el arte como eje central de la acción social, trabajando desde la sostenibilidad, los preceptos de la economía feminista y recuperando oficios desde la tradición, la práctica artística y la contemporaneidad. Potencian el uso de metodologías comprometidas como el Aprendizaje-Servicio evidenciando la necesidad de la participación ciudadana en la formación universitaria.

Por otro lado, está el alumnado de tercero de la ESO de los Institutos de Educación Secundaria Rovira-Forns y Estela Ibèrica de Santa Perpètua de Mogoda que participa de la experiencia mediante la actividad curricular de Servicio Comunitario. Cerca de veinte alumnos y alumnas han participado directamente en el proyecto entre los dos centros.

El Servicio de Educación del Ayuntamiento de Santa Perpètua de Mogoda, en el marco del Proyecto Educativo de Ciudad (PEC), coordina y gestiona las diferentes actividades relacionadas con el Servicio Comunitario y actúa de mediador entre los diferentes agentes implicados.

Por último, los equipamientos culturales del municipio ofrecen el espacio de encuentro y de exposición del proceso y de los resultados, promoviendo las interacciones y colaborando en la difusión de la experiencia. Participan el Departamento de Cultura del Ayuntamiento, el Museo Municipal, el espacio de la Granja Soldevila y la Biblioteca Municipal Josep Jardí.

\subsection{Identitats truncades. Una ficha pedagógica para provocar la creatividad}

El proyecto se articula principalmente a partir de una ficha pedagógica siguiendo los preceptos de las metodologías del descubrir. En este caso, se acompaña del soporte formativo videográfico Extreure patrons (Grau Costa, 2020) debido a la imposibilidad de impartir la formación presencialmente en la primera parte del proyecto dada la situación crítica provocada por la pandemia del Covid-19. El video muestra el procedimiento de extracción de patrones de una pieza textil. Se acompaña de unas frases insertadas que aportan los elementos clave para seguir el procedimiento y asimilar el léxico específico que necesita el alumnado. 


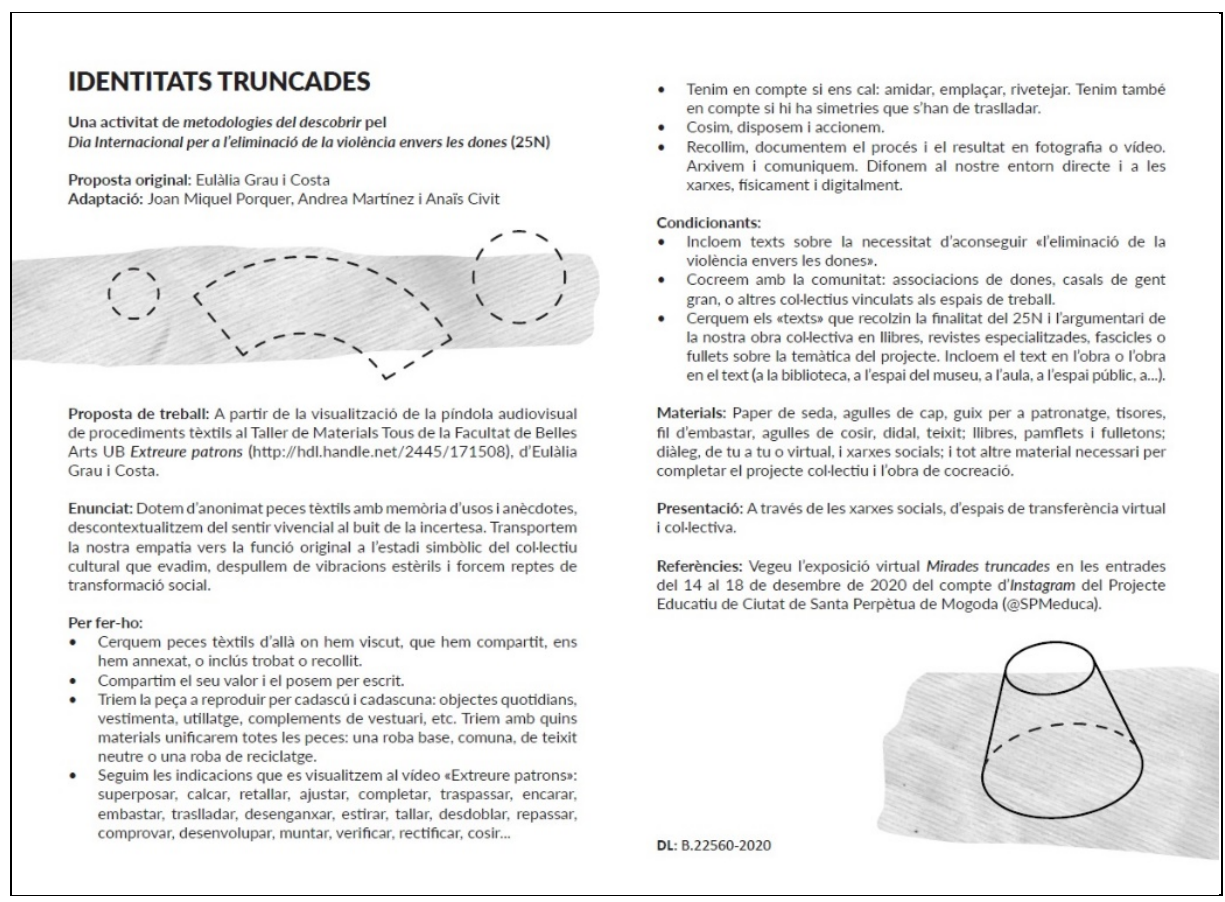

Figura 1. Ficha pedagógica Identitats truncades

La ficha pedagógica apela al alumnado marcando unas pautas procedimentales y una temática aparentemente muy concretas, pero con un lenguaje y una construcción que generan interrogantes y están sujetas a interpretación. De esta manera, se plantea un encargo que el alumnado acepta como asumible pero que requiere desarrollar estrategias propias y una reflexión constante para poder resolver el encargo. Así el enunciado de la ficha nos dice:

"Dotemos de anonimato piezas textiles con memoria de usos y anécdotas, descontextualicemos del sentir vivencial al vacio de la incertidumbre. Transportemos nuestra empatía hacia la función original en el estadio simbólico del colectivo cultural que evadimos, desnudemos de vibraciones estériles y forcemos retos de transformación social" (Grau Costa et al., 2020).

Ello va seguido de las pautas del encargo que podríamos resumir en: escoger una pieza textil que tenga algún significado para el alumnado, compartir el significado, escoger el material textil con el que reproducirlo y vincular el objeto con el nuevo significado en relación con la temática común del encargo, la eliminación de la violencia hacia las mujeres. La temática se vincula con la fecha del 25 de noviembre, Día Internacional de la Eliminación de la Violencia contra la Mujer, pero se ampara en un marco más amplio para reflexionar acerca de cuestiones de género, de identidad y de economía feminista. Por último, se impone la necesidad de reflejar por escrito parte de las reflexiones implícitas en la pieza para acompañar a esta en la exposición.

\subsection{Desarrollo del proyecto}

Se trata de un proyecto anual, en dos fases, ligado al calendario del curso escolar 2020/21 tanto universitario como de Educación Secundaria. Una primera fase con el Instituto Rovira-Forns de octubre de 2020 a febrero de 2021 y una segunda fase con el Instituto Estela Ibèrica de febrero a mayo de 2021. Ambas dentro del horario semanal 
de los dos Institutos dedicado al Servicio Comunitario y del horario de la asignatura UEA: Nous Recobriments $i$ Continents en art del Grado mediante Aprendizaje-Servicio.

\subsubsection{Secuencia didáctica}

Se plantea una secuencia didáctica diseñada para poder ser desarrollada en unas seis $\mathrm{u}$ ocho sesiones de una hora y media de trabajo.

Primera y segunda sesión: Lectura individual y posteriormente colectiva de la ficha Identitats truncades. Visionado de la píldora videográfica formativa Extreure Patrons. Asignación del material individual $(100 \times 150 \mathrm{~cm}$ de tela de algodón y lino blanca y varios pliegos de papel de Manila). Muestra de libros de costura y patronaje antiguos. Puesta en común de las nociones previas sobre costura, antecedentes familiares y concepciones asociadas a la tradición textil. Debate sobre el papel de las mujeres en relación con las tareas textiles y de cuidado. Planteamiento de los objetivos de trabajo y expositivos del proyecto, así como una primera orientación acerca del objeto escogido para la extracción del patrón.

Tercera sesión: Puesta en común de las reflexiones individuales en torno a los objetos escogidos. Relación del objeto, el material y el nuevo valor simbólico y sus posibles derivas. Asesoramiento y extracción de patrones del objeto elegido.

Cuarta, quinta y sexta sesión: Sesiones se seguimiento. Traspaso del patrón a la tela y proceso de costura de la pieza definitiva.

Sexta y séptima sesión: Presentación de la pieza final y planificación expositiva. Se requiere al alumnado que exponga la pieza teniendo en cuenta el espacio y la composición. Además de acompañarla de título y de un breve texto reflexivo, descriptivo o alegórico.

\subsubsection{Aplicación de la secuencia}

Es necesario dejar constancia de las diferencias y similitudes en la aplicación de la secuencia didáctica del proyecto en cada uno de los dos institutos.

La primera fase del proyecto se desarrolló en siete sesiones, con un total de nueve alumnos y alumnas del IES Rovira-Forns y cuatro alumnas de la Facultad de Bellas Artes. Es necesario comentar que la situación de confinamiento provocada por el Covid19 tuvo incidencia en esta primera fase del desarrollo del proyecto por lo que solamente se pudo realizar una primera sesión formativa presencial en el Museo Municipal de la población. No obstante, esta sesión fue clave para poder tomar el primer contacto con el alumnado participante, asentar las bases de la tarea, facilitar el material y provocar muchos interrogantes para confrontar y resolver de manera individual y colectiva con sus compañeros y familiares. El resto del proyecto se realizó y tutorizó de manera virtual por completo con los retos que esto supone. En esta primera fase establecimos la fecha del 25 de noviembre, Día Internacional de la Eliminación de la Violencia contra la Mujer, como objetivo expositivo.

La segunda fase del proyecto se desarrolló en nueve sesiones, con un total de nueve alumnos y alumnas del IES Estela Ibèrica, cuatro alumnas de la Facultad de Bellas Artes como tutoras y tres alumnas más para realizar el montaje de la exposición final. Solamente una de ellas tenía continuidad desde la primera fase del proyecto. La mejoría de la situación causada por el Covid-19 permitió desarrollar las sesiones en su mayor 
parte de manera presencial. En esta ocasión, se plantearon las fechas del 8 de marzo, Día Internacional de la Mujer, y del 23 de abril, Diada de Sant Jordi o Dia del Libro, como fechas expositivas. El proyecto finalizó el día 15 de mayo con el acto de clausura de la exposición.

Es importante destacar que el alumnado del Grado que tutoriza al de Secundaria, está realizando paralelamente como parte de su formación académica el mismo encargo. Veremos pues, los puntos de convergencia y diferencias entre los resultados.

\subsection{Resultados}

Primeramente, hablaremos de las creaciones artísticas del alumnado, los resultados expositivos y de difusión del proyecto.

Es necesario dejar constancia de las diferencias producidas entre uno y otro grupo de alumnado. Si bien es cierto que los condicionantes del desarrollo del proceso de trabajo no han sido los mismos, también es cierto que los grupos de trabajo no se comportan nunca de la misma manera. Proceden de centros formativos distintos, con una cohesión de grupo, un nivel de madurez y capacidad de adaptación diferente. El IES RoviraForns tiene una amplia experiencia en el trabajo por proyectos, por lo que su alumnado ha asimilado más fácilmente el tipo de encargo y la autonomía de trabajo que requería la tarea aún sin disponer de las facilidades de herramientas y del apoyo que facilita la presencialidad. Por otro lado, el alumnado del IES Estela Ibèrica, ha preferido trabajar constantemente en grupo y desarrollar piezas colectivas, produciéndose en ocasiones desequilibrios de implicación, de trabajo horizontal y cierta dependencia hacia la figura de las tutoras. Aún con las diferencias en el comportamiento de los dos grupos, ambos consiguieron resolver de manera satisfactoria el encargo y mostraron un nivel parecido de compromiso con el proyecto.

De esta manera, el alumnado del IES Rovira-Forns realizó piezas de manera individual tratando cuestiones relativas a la violencia de género, el derecho a la intimidad, el miedo o el aislamiento derivado del Covid-19. Destacamos el trabajo de Iván Manzano Importancia a l'interior que realizó un poncho en el que escribió palabras despectivas y relativas al abuso en la parte exterior de la tela y palabras positivas acompañadas del símbolo de un corazón en el interior para hablar sobre el derecho a la intimidad diciéndonos que "las cosas más interesantes de ti están en tu interior, aunque los demás te digan cosas malas". Destaca también el trabajo de Jaume Umbert La por a la foscor que extrajo el patrón a un muñeco de trapo que tenía de pequeño y realizó un nuevo muñeco con tela negra, ayudado por su abuela, para hablar del miedo, especialmente el miedo a la oscuridad, y a lo desconocido.

El alumnado del IES Estela-Ibèrica realizó dos instalaciones de manera conjunta. En esta ocasión quisieron reflexionar alrededor de la identidad sexual y de género. La primera pieza parte de una vivencia de su propia población en la que en un centro educativo se designó un baño separado solamente para uso de un compañero transgénero. Ésta "solución” indignó al alumnado del centro y provocó un debate acerca de la cuestión. Por ello, decidieron realizar una instalación de un baño elaborando una simbología propia acompañada de un rollo de fragmentos textiles, emulando un papel higiénico, en la que recoger frases discriminatorias y estereotipos que los propios visitantes podían escribir durante la exposición. Por otro lado, construyeron las barras de las banderas transgénero y bisexual a partir del patrón de unas espadas láser. Si bien 
esta idea generó bastante debate entre el alumnado y las tutoras, fue un motivo perfecto para hablar del proceso de construcción de la obra de arte, de la simbología y la percepción del espectador: significados fálicos, estereotipos de género masculino vinculados al poder, a la violencia e incluso a la ausencia de personajes femeninos en ámbitos tradicionalmente masculinos como son los videojuegos.

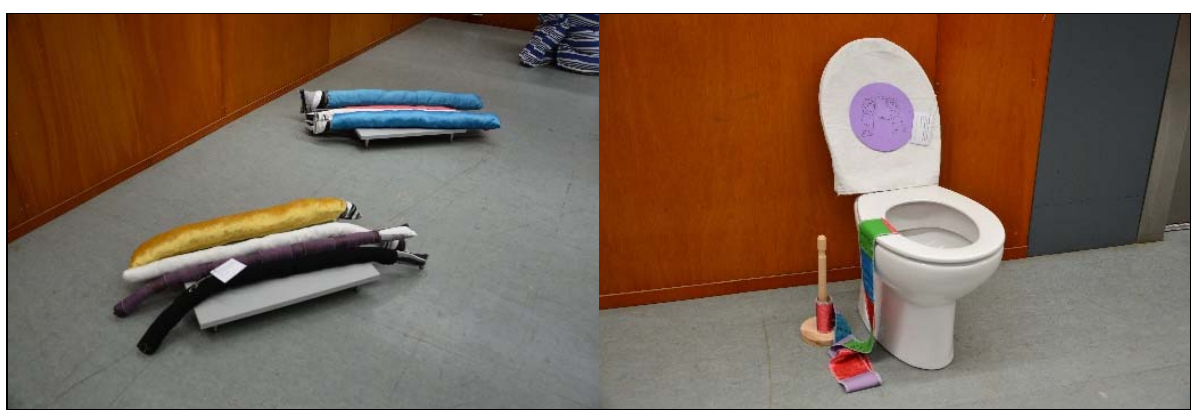

Figura 2. Obras del alumnado del IES Estela Ibèrica. Fuente: Anaïs Civit

El alumnado y profesorado de la Facultad de Bellas Artes realizó piezas durante todo el proyecto compartiendo el proceso y debatiendo la elaboración con el alumnado. Destacan Fins als mugrons! de Júlia Cadevall que extrae el patrón de un bidón de gasolina para transformarlo en un bidón textil lila con un mensaje combativo o Anar a conjunt de la profesora Andrea Martínez que extrae el patrón de una silla para evocar ese espacio de costura de su madre cuando era pequeña.

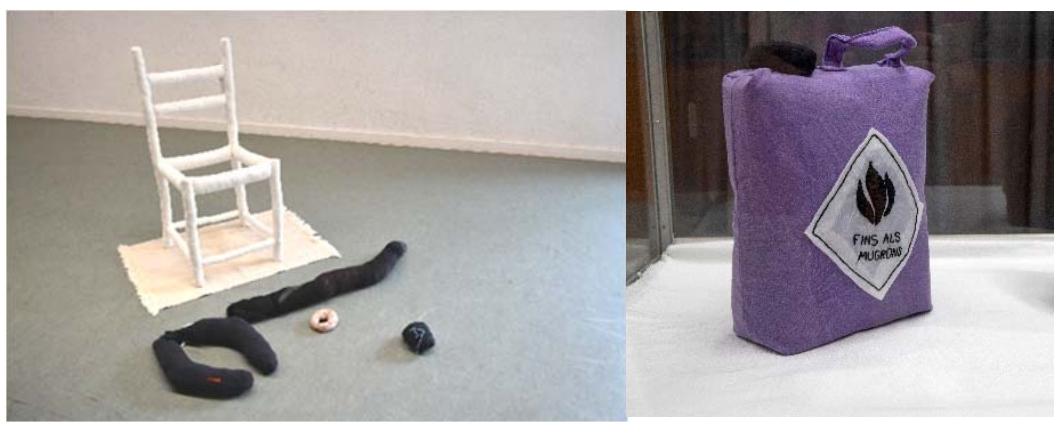

Figura 3. Obras Anar a conjunt de Andrea Martínez y Fins als mugrons! de Júlia Cadevall. Fuente: Anaïs Civit y Gala Espin

A nivel expositivo, se realizó una primera exposición virtual Mirades truncades a través de la cuenta de Instagram de Santa Perpètua Educa (@SPMeduca) del 14 al 18 de febrero de 2021 en el que el alumnado del IES Rovira-Forns tuvo la oportunidad de exponer sus piezas. El alumnado realizó un montaje expositivo fotográfico de su pieza y lo acompañó de un texto. De este modo, se trabajaba la competencia lingüística y la reflexión en torno a la propia creación. Recogemos a continuación uno de los textos de la alumna Jana Valle que acompaña a su pieza $A$ les $18 \mathrm{~h}$ a casa es fa de nit:

"Mi camiseta representa nuestra sociedad actual llena de violencia de género, así como el trato que recibimos las mujeres y que muchas veces callamos. He tratado de plasmar mi idea a través de un texto obtenido de un cartel de una manifestación feminista. Este texto (De camino a casa quiero ser libre, no valiente) representa cómo nosotras las mujeres ya nos hemos cansado de tener que ser valientes al salir a la calle. Queremos ser completamente libres de salir con cualquier ropa o a cualquier hora sin tener miedo de si llegaremos sanas y 
salvas a casa. Además, he enfatizado la palabra "libre" con color lila que representa el color de la mujer".

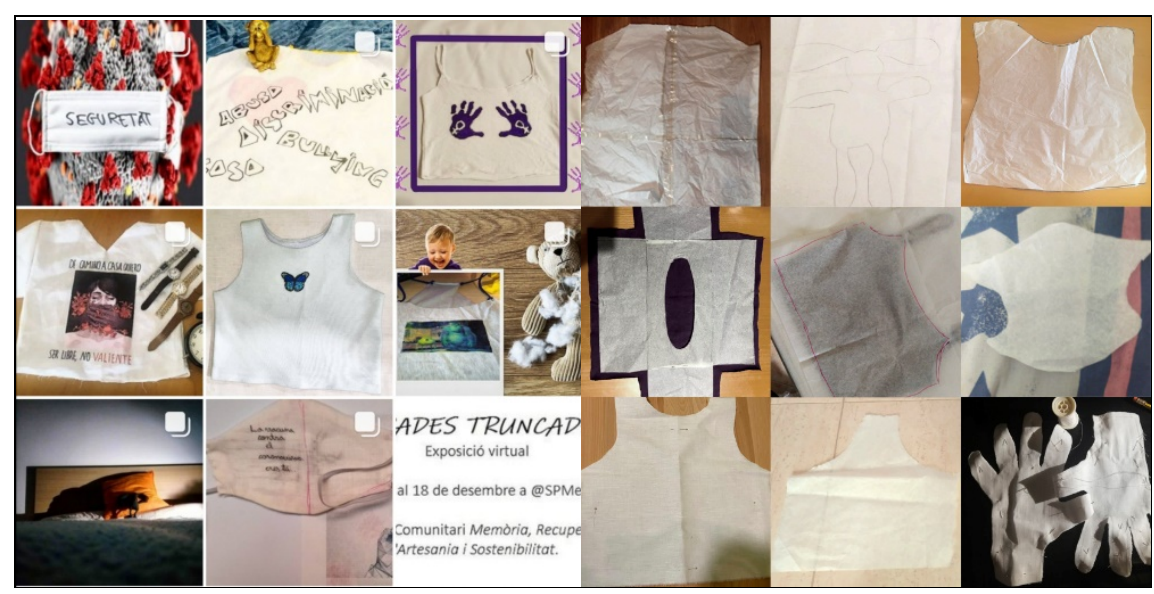

Figura 4. Detalles de las obras y los patrones de La seguretat és la salvació de la pandemia de Arnau Garcia; La felicitat i la tristesa de Carlota Martínez; Importància a l'interior, de Iván Manzano; A les 18 hores a casa es fa de nit de Jana Valle; La por a la foscor de Jaume Umbert; Violència de gènere de Judit Guirado; Covid vs Humanitat de Yahia Benslimam; e Infància digna de Mireia Garcia. Fuente: Propios autores

Finalmente, el proyecto confluye en una exposición final, Identitats truncades: Sentits $i$ Serveis, en el Museo Municipal, Granja Soldevila, del municipio de Santa Perpètua de Mogoda entre el 23 de abril y el 15 de mayo de 2021. La exposición reúne las obras de todos y todas las participantes del proyecto y se acompaña de las Jornadas de Investigación y Docencia artística Rellegint Identitats en las que se realiza el procedimiento tradicional en desuso de batear la lana -empleado para airear la lana para rellenar los colchones- como actividad vertebradora. Colectivamente rellenan, en este caso, las "espadas láser" textiles que han realizado durante el proyecto, así como calcetines y medias estropeadas, para contribuir a la exposición.

Referente a la difusión del proyecto, se publicaron un artículo (L'Informatiu Santa Perpètua de Mogoda, 2021a) y una noticia (L'Informatiu Santa Perpètua de Mogoda, 2021b) en el periódico local de Santa Perpètua de Mogoda, l'Informatiu. En el artículo se recoge la experiencia vivida por el alumnado de los Institutos y el alumnado y profesorado de la Facultad de Bellas Artes. De esta manera se consigue dar a conocer el proyecto a nivel local y llegar a todos aquellos colectivos que no acceden a la información por los medios digitales.

\subsection{Beneficios pedagógicos}

Referente a los beneficios pedagógicos, cabe decir que en este proyecto el alumnado es el centro del aprendizaje en un doble sentido. Por un lado, los alumnos de los Institutos son quienes realizan las creaciones artísticas que posteriormente se expondrán. Por otro lado, el alumnado del grado de Bellas Artes se encarga de tutorizar el trabajo del alumnado del Instituto, a la vez que recibe la formación indicada y colaboran en la creación de las piezas artísticas. El alumnado, pues, es el centro y el motor del proyecto siendo creadores y tutores a la vez. Este aspecto fomenta la autonomía de trabajo entre los participantes del proyecto y permite que la importancia recaiga en el proceso de creación y no en el resultado final. La propuesta, además, 
permite a la Universidad salir de su recinto y situarse en el entorno social del territorio cercano, en la Educación Secundaria y en la realidad de los métodos de producción populares. Además, cabe decir que la exposición de los resultados permite a los participantes del proyecto trabajar con el objetivo de resolver un problema dado.

El alumnado de Bellas Artes adquiere competencias que se relacionan con las capacidades de expresión y transmisión de conocimientos, así como la capacidad de supervisar y tutorizar proyectos de creación artística. Mientras que el alumnado de los institutos adquiere capacidades y competencias relacionadas con la autonomía de trabajo, la expresión artística y la resolución de problemas.

Consideramos que el proyecto es un modelo de éxito y puede suponer un modelo que puede seguirse por parte de otros centros educativos a la hora de incorporar propuestas de creación artística con mirada comunitaria en actividades curriculares, así como establecer colaboraciones con agentes culturales que permitan la salida del alumnado de las aulas. La propuesta, pues, evidencia la necesidad urgente de recuperar las memorias locales, los saberes populares y los oficios y profesiones que corren el riesgo de caer en el olvido, especialmente el artesanado textil, fuertemente vinculado a la historia de las mujeres y al ámbito doméstico. En este sentido, el proyecto defiende y recupera el uso del léxico y la terminología técnica en lengua catalana, reincorporándolo a la sociedad en forma de vehículo de expresión artística, altavoz de las reivindicaciones de género y herramienta de educación en valores.

El proyecto conecta los jóvenes con el territorio y sus necesidades, fomentando la conciencia social y adquiriendo nuevos mecanismos de expresión mediante lenguajes visuales y plásticos, dotándolos de una función vehicular en los procesos de dinamización cultural y en las actividades de intervención socio-comunitaria. Así, se revalorizan los oficios y las tradiciones textiles en el contexto cotidiano, dotándolas de una importancia que conecte con los jóvenes de la Universidad y de los centros de Educación Secundaria.

\section{CONCLUSIONES}

Mediante la metodología del Aprendizaje-Servicio no solamente el alumnado que participa del Servicio Comunitario realiza la tarea, sino que desarrolla el encargo a la vez que sus tutoras y el equipo docente universitario implicado en el proyecto. De este modo se consigue un compromiso común, se diluyen las jerarquías, se produce una transferencia de conocimiento constante y se producen creaciones artísticas en las que cada participante puede trabajar sus potencialidades. Además, se genera un entorno seguro de debate en el que dar salida a las inquietudes y problemáticas que les conciernen (cuestiones de violencia e identidad sexual y de género, sostenibilidad, etc.) a través del lenguaje artístico para comunicar cuestiones que a menudo cuesta verbalizar y visibilizar.

El alumnado de Secundaria trabaja mediante procedimientos esenciales de la práctica artística con una perspectiva comunitaria y expone los resultados considerando sus creaciones dentro de la categoría de arte. Integran a la comunidad en la realización de las piezas, así como en las actividades de difusión del proceso y los resultados fomentando las relaciones intergeneracionales. Se recuperan, pues, procesos productivos propios de la tradición textil y artesanal, dando valor a la memoria histórica 
local y a todos aquellos métodos de elaboración que se encuentran en desuso o incluso se están perdiendo.

El proyecto ha demostrado ser un modelo de éxito con capacidad para ser exportado a otros contextos. La pandemia del Covid-19 sin duda ha obligado a reconfigurar numerosos aspectos que bien seguro podrán readaptarse en un futuro cercano. Aun así, se trata de una propuesta exitosa con amplios beneficios para el alumnado participante, tanto perteneciente a la Universidad como a los Institutos de Educación Secundaria, y con una prometedora capacidad para ampliar el marco de enseñanza y aprendizaje a un entorno alejado del aula y ofrecer al alumnado nuevos lenguajes para comunicar y transformar problemáticas que les afectan directamente y forman parte de sus inquietudes diarias.

\section{BIBLIOGRAFIA}

Batlle, R. (Coord.) (2015) Avaluació dels aprenentatges en els projectes d'AprenentatgeServei. Fundació Jaume Bofill. Recuperado de:

https://aprenentatgeservei.cat/wp-content/uploads/guies/aps_avaluacio_aprenentatge-ambannex.pdf

Carrasco, C. (2014). Con voz propia. La economía feminista como apuesta teórica y política. La Oveja Roja.

Cortázar Martínez, Coronel Olivares, C., Escalante Lozada, A. y González Ramírez, C. (2014). Contaminación generada por colorantesde la industria textil. Vida Cientifica. Boletín Científico De La Escuela Preparatoria No4, 2(3), 8-12. Recuperado de:

https://repository.uaeh.edu.mx/revistas/index.php/prepa4/article/download/1862/5730?inli $n e=1$

Deeley, S. J. (2016). El Aprendizaje-Servicio en educación superior. Teoría, práctica y perspectiva crítica. Narcea.

Escofet, A y Rubio, L. (2017) Aprendizaje-servicio (ApS): claves para su desarrollo en la Universidad. Octaedro.

Giralt, E.G. (2009). La carpeta de aprendizaje qué, cómo y por qué. Observar, 3, 55-88.

Grau Costa, E. (Coord.) (2009). Expogenealogies femenines en la pràctica artística. Facultat de Belles Arts de la Universitat de Barcelona.

Grau Costa, E. (Coord.) (2013). Dimensions XX. Genealogies Femenines. Art, recerca i docència. Volum 1. Edicions Saragossa y Comissió de publicacions i edicions del Departament d'Escultura UB.

Grau Costa, E. (2020). Extreure patrons. Píndoles formatives audiovisuals del Taller de Materials Tous a la Facultat de Belles Arts UB. Dipòsit Digital UB. Recuperado de: http://hdl.handle.net/2445/171508

Grau Costa, E. y Porquer Rigo, J.M. (Eds.) (2017). Dimensiones XX. Genealogias de anonimato. Arte, investigación y docencia. Volumen 2. Edicions Saragossa y Sección de Escultura y Creación del Departamento de Artes y Conservación-Restauración de la Universitat de Barcelona.

Grau Costa, E., Porquer Rigo, J.M., Martínez, A. y Civit, A. (2020). Identitats truncades. Una activitat de metodologies del descobrir per a l'eliminació de la violència envers les dones. Universitat de Barcelona

L'Informatiu Santa Perpètua de Mogoda (2021a). Expressar emocions a través de peces de roba. L'Informatiu Santa Perpètua de Mogoda, 655, 17.

http://staperpetua.cat/menu-principal/serveis/presidencia-i-serveis-generals/comunicacio1/fitxers-pdf-de-linformatiu/any-2021/linformatiu_655.pdf/view 
L'Informatiu Santa Perpètua de Mogoda (2021b). Identitats truncades, una mostra amb visió de gènere. L'Informatiu Santa Perpètua de Mogoda, 659, 31.

http://staperpetua.cat/menu-principal/serveis/presidencia-i-serveis-generals/comunicacio1/fitxers-pdf-de-linformatiu/any-2021/linformatiu_659.pdf/view

Menéndez-Varela, J.L. y Grigori-Giralt, E. (2017). La construcción de la identidad profesional de estudiantes universitarios de arte a través de proyectos de aprendizaje-servicio. Arte, Individuo y Sociedad, 29(3), 417-443. https://doi.org/10.5209/ARIS.55249

Moretó Alvarado, L. y Gregori, E. (2014). Imatges fotogràfiques com a mitjà de relació entre exreclusos i societat. Una proposta d'aprenentatge-servei per a les arts. Observar, 8, 5770. Recuperado de: https://raco.cat/index.php/Observar/article/view/286835

Porquer Rigo, J.M., Grau Costa, E., Ros Vallverdú, J. y Asensio Fernández, J. A. (2015). Lea las instrucciones de uso. Fichas de trabajo contemporáneas para una asignatura introductoria a los procesos y proyectos de creación artística en 3rd International Congress of Educational Sciences and Developement, 570. ICESD.

Porquer Rigo, J.M., Grau Costa, E. y Asensio Fernández, J. A. (2020). Transferència activa: Aprenentatge Servei en el context de la Facultat de Belles Arts de la Universitat de Barcelona. En Jeffrey Swartz (Ed.), Actes del Congrés sobre la recerca en la pràctica de les arts, (pp. 221-231). Universitat de Barcelona - Campus de les Arts.

Puig, J. (Coord.). (2012). Compromís cívic i aprenentatge a la universitat: Experiències $i$ institucionalització de l'aprenentatge servei. Graó.

Puleo, A. (2011). Ecofeminismo para otro mundo posible. Cátedra.

Rubio Serrano, L., Moliner Miravet, L. y Francisco Amat, A. (Coord.) (2014) Construyendo ciudadanía crítica y activa. Experiencias s/ el aprendizaje servicio en las universidades del Estado Español. Icaria.

Santos Rego, M.A., Sotelino Losada, A. y Lorenzo Moledo, M. (2016). El aprendizaje-servicio en la Educación Superior: Una vía de innovación y de compromiso social. Revista Educación y Diversidad, 10(2), 17-24.

Sasiain Camarero-Núñez, A. (2012). Una Herida Arropada: Reminiscencias del vestido en la escultura contemporánea. Una apuesta pedagógica desde la práctica artística. [Tesis doctoral, Universitat de Barcelona].

Uruñuela, P.M. (2018). La metodología del Aprendizaje-Servicio. Aprender mejorando el mundo. Narcea Ediciones. 